\title{
A Numerical Computation of a System of Linear and Nonlinear Time Dependent Partial Differential Equations Using Reduced Differential Transform Method
}

\author{
Brajesh Kumar Singh and Mahendra \\ Department of Applied Mathematics, School for Physical Sciences, Babasaheb Bhimrao Ambedkar University, Lucknow, \\ Uttar Pradesh 226025, India
}

Correspondence should be addressed to Brajesh Kumar Singh; bksingh0584@gmail.com

Received 20 April 2016; Revised 18 September 2016; Accepted 4 October 2016

Academic Editor: Davood D. Ganji

Copyright (C) 2016 B. K. Singh and Mahendra. This is an open access article distributed under the Creative Commons Attribution License, which permits unrestricted use, distribution, and reproduction in any medium, provided the original work is properly cited.

\begin{abstract}
This paper deals with an analytical solution of an initial value system of time dependent linear and nonlinear partial differential equations by implementing reduced differential transform (RDT) method. The effectiveness and the convergence of RDT method are tested by means of five test problems, which indicates the validity and great potential of the reduced differential transform method for solving system of partial differential equations.
\end{abstract}

\section{Introduction}

The reduced differential transform method has been successfully employed to solve various types of linear and nonlinear, homogeneous or nonhomogeneous, equations appearing in science and engineering. Partial differential equations have also been applied in modeling many physical engineering problems and differential equations in nonlinear dynamics [1-3]. Most of the partial differential equations cannot be solved exactly, and so, developing schemes for getting accurate and efficient numerical solution differential equations have been an active research area. Burgers' equation [4], a system of nonlinear fractional differential equations [1], and nonlinear Klein-Gordon equation with a quadratic nonlinear term [2] have been solved using Adomian decomposition method. A system of nonlinear fractional partial differential equations has been solved using homotopy analysis method by Jafari and Seifi [3] and Bataineh et al. [5], using variational iteration method by Wazwaz [6]. In [7], Wang and Cheng adopted variational method and finite element approach to solve damped nonlinear Klein-Gordon equations.

The coupled Burgers equation has been solved by using various schemes; among them are variational iteration method [8], Adomian-Pade technique [9], fourth-order compact schemes [10], a composite numerical scheme [11], lattice Boltzmann method [12], finite element and finite difference method [13], two algorithms based on cubic spline function technique [14], a robust finite difference scheme [15], and modified extended cubic $\mathrm{B}$-spline differential quadrature method [16]; while using modified cubic B-spline differential quadrature method Burgers' and Burgers-Huxley equations have been solved in [17-19], respectively. The fractional models of Burgers equation have been solved in [19-22]. The space- and time-fractional coupled Burgers equations have been solved using generalized differential transform method [19] and homotopy perturbation method [21]. Reduced differential transform method is used to solve $(1+n)$-dimensional Burgers' equation [20]. Recently, Prakash et al. [22] adopted fractional variational iteration method to solve fractional coupled Burgers equations.

Keskin and Oturanç [23] have developed reduced differential transform method to solve partial differential equations of integer order [24] as well as fractional order. After Keskin and Oturanç, RDT method has been implemented for the numerical computation of various physical models of engineering and sciences [25-27]. 
The main goal of this paper is to provide an analytical solution of initial value system of time dependent partial differential equations obtained by employing RDT method developed by Keskin and Oturanç [23].

\section{Reduced Differential Transform Method}

The basic properties of the fractional reduced differential transform method are described in this section. Let $\psi(x, t)$ be a function of two variables such that $\psi(x, t)=f(x) g(t)$. By using the properties of the one-dimensional differential transform (DT) method $\psi(x, t)$ can be written as

$$
\psi(x, t)=\sum_{i=0}^{\infty} f(i) x^{i} \sum_{j=0}^{\infty} g(j) t^{j}=\sum_{i=0}^{\infty} \sum_{j=0}^{\infty} \Psi(i, j) x^{i} t^{j},
$$

where $\Psi(i, j)$ is referred to as the spectrum of $\psi(x, t)$ and is defined by

$$
\Psi(k, h)=\frac{1}{h ! k !}\left(\frac{\partial^{k+h} \psi(x, t)}{\partial x^{k} \partial t^{h}}\right)_{\left(x_{0}, t_{0}\right)}
$$

For more details on DT method, see [28] and the references therein.

Denote the lowercase $\psi(x, t)$ as the original function while its fractional reduced transformed function is denoted by the uppercase $\Psi_{k}(x)$.

Definition 1. If $\psi(x, t)$ is analytic and continuously differentiable with respect to $x$ and $t$, then RDT of $\psi$ is given by

$$
U_{k}(x)=\frac{1}{k !}\left[\frac{\partial^{k} u(x, t)}{\partial t^{k}}\right]_{t=0}
$$

The reduced inverse differential transform of $U_{k}(x)$ is defined as follows:

$$
u(x, t)=\sum_{k=0}^{\infty} U_{k}(x) t^{k}
$$

Equations (3) and (4) together reduce to

$$
u(x, t)=\sum_{k=0}^{\infty} \frac{1}{k !}\left(\frac{\partial^{k} u(x, t)}{\partial t^{k}}\right)_{t=0} t^{k}
$$

The basic properties of RDT method are found in $[1,4]$ and can be deduced from (3) and (4), given in the following.
2.1. Some Basic Properties and Notation of RDT Method. In this section, the properties of RDT method as in [23-25] have been revisited to complete our study.

Property 1. If $w(x, t)=u(x, t) \pm v(x, t)$, then $W_{k}(x)=U_{K}(x) \pm$ $V_{k}(x)$.

Property 2. If $w(x, t)=\alpha u(x, t)$, then $W_{k}(x)=\alpha U_{k}(x)$.

Property 3. If $w(x, t)=\left[x^{m} t^{n}\right]$, then $W_{k}(x)=x^{m} \delta(k-n)$, where

$$
\delta(k-n)= \begin{cases}1, & \text { when } k=n \\ 0, & \text { when } k \neq n\end{cases}
$$

Property 4. If $w(x, t)=\left[x^{m} t^{n} u(x, t)\right]$, then $W_{k}(x)=$ $x^{m} U_{k-n}(x)$.

Property 5. If $w(x, t)=\left[\partial^{r} u(x, t) / \partial t^{r}\right]$, then $W_{k}(x)=(k+$ 1) $(k+2) \cdots(k+r) U_{k+r}(x)=((k+r) ! / k !) U_{k+r}(x)$.

Property 6. If $w(x, t)=[\partial u(x, t) / \partial x]$, then $W_{k}(x)=\partial U_{k}(x) /$ $\partial x$.

Property 7. If $w(x, t)=u(x, t) v(x, t)$, then $W_{k}(x)=$ $\sum_{i=0}^{k} U_{i}(x) V_{k-i}(x)$.

Property 8. If $w(x, t)=[u(x, t)]^{m}$, then

$$
W_{k}(x)= \begin{cases}U_{0}(x), & k=0 \\ \sum_{n=1}^{k} \frac{(m+1) n-k}{k U_{0}(x)} U_{n}(x) W_{k-n}(x), & k \geq 1\end{cases}
$$

For details on RDT method we refer the readers to [23-25].

\section{Results and Discussion}

In this section, we give five test problems of linear and nonlinear partial differential equations (PDEs) using reduced differential transform (RDT) method.

Example 2. Consider the initial value system of linear PDEs:

$$
\begin{aligned}
\frac{\partial u(x, t)}{\partial t} & =\frac{\partial v(x, t)}{\partial x}-v(x, t)-u(x, t), \\
\frac{\partial v(x, t)}{\partial t} & =\frac{\partial u(x, t)}{\partial x}-v(x, t)-u(x, t), \\
u(x, 0) & =\sinh (x), \\
v(x, 0) & =\cosh (x) .
\end{aligned}
$$


On using RDT method (8) reduces to a set of recurrence relations as follows:

$$
\begin{aligned}
(1+k) U_{k+1}(x) & =\frac{\partial}{\partial x} V_{k}(x)-V_{k}(x)-U_{k}(x), \\
(1+k) V_{k+1}(x) & =\frac{\partial}{\partial x} U_{k}(x)-V_{k}(x)-U_{k}(x), \\
U_{0}(x) & =\sinh (x) \\
V_{0}(x) & =\cosh (x) .
\end{aligned}
$$

On solving system (9), we get

$$
\begin{aligned}
U_{2 i+1}(x) & =\frac{-1}{(2 i+1) !} \cosh (x) ; \\
U_{2 i}(x) & =\frac{1}{2 i !} \sinh (x), \\
V_{2 i+1}(x) & =\frac{-1}{(2 i+1) !} \sinh (x) ; \\
V_{2 i}(x) & =\frac{1}{2 i !} \cosh (x),
\end{aligned}
$$

$$
i=0,1,2, \ldots
$$

Using inverse RDT method (4), we get

$$
\begin{aligned}
& u(x, t)=\sinh (x-t), \\
& v(x, t)=\cosh (x-t) .
\end{aligned}
$$

The same solution is obtained by using homotopy analysis method [3], variational iteration method [6], and homotopy perturbation method [21]. The solution behavior of $u, v$ is depicted in Figure 1.

Example 3. Consider the following initial value system of nonlinear PDEs:

$$
\begin{aligned}
\frac{\partial u}{\partial t}+\left(\frac{\partial v}{\partial x}\right)\left(\frac{\partial w}{\partial y}\right)-\left(\frac{\partial v}{\partial y}\right)\left(\frac{\partial w}{\partial x}\right) & =-u, \\
\frac{\partial v}{\partial t}+\left(\frac{\partial u}{\partial x}\right)\left(\frac{\partial w}{\partial y}\right)-\left(\frac{\partial u}{\partial y}\right)\left(\frac{\partial w}{\partial x}\right) & =v, \\
\frac{\partial w}{\partial t}+\left(\frac{\partial u}{\partial x}\right)\left(\frac{\partial v}{\partial y}\right)-\left(\frac{\partial u}{\partial y}\right)\left(\frac{\partial v}{\partial x}\right) & =w, \\
u(x, y, 0) & =e^{x+y}, \\
v(x, y, 0) & =e^{x-y} \\
w(x, y, 0) & =e^{-x+y} .
\end{aligned}
$$

On using RDT method (12) reduces to a set of recurrence relations as follows:

$$
\begin{aligned}
& (1+k) U_{k+1}(x, y)=-U_{k}-\sum_{i=0}^{k}\left\{\left(\frac{\partial}{\partial x} V_{i}\right)\left(\frac{\partial}{\partial y} W_{k-i}\right)\right. \\
& \left.-\left(\frac{\partial}{\partial y} V_{i}\right)\left(\frac{\partial}{\partial x} W_{k-i}\right)\right\}, \\
& (1+k) V_{k+1}(x, y)=V_{k}-\sum_{i=0}^{k}\left\{\left(\frac{\partial}{\partial x} U_{i}\right)\left(\frac{\partial}{\partial y} W_{k-i}\right)\right. \\
& \left.-\left(\frac{\partial}{\partial y} U_{i}\right)\left(\frac{\partial}{\partial x} W_{k-i}\right)\right\}, \\
& (1+k) W_{k+1}(x, y)=W_{k}-\sum_{i=0}^{k}\left\{\left(\frac{\partial}{\partial x} U_{i}\right)\left(\frac{\partial}{\partial y} V_{k-i}\right)\right. \\
& \left.-\left(\frac{\partial}{\partial y} U_{i}\right)\left(\frac{\partial}{\partial x} V_{k-i}\right)\right\}, \\
& U_{0}(x, y)=e^{x+y}, \\
& V_{0}(x, y)=e^{x-y}, \\
& W_{0}(x, y)=e^{-x+y} .
\end{aligned}
$$

On solving system (13), we have

$$
\begin{aligned}
U_{1}(x, y) & =\frac{(-1)}{1 !} e^{x+y}, \\
V_{1}(x, y) & =\frac{1}{1 !} e^{x-y} \\
W_{1}(x, y) & =\frac{1}{1 !} e^{-x+y}, \\
U_{2}(x, y) & =\frac{(-1)^{2}}{2 !} e^{x+y}, \\
V_{2}(x, y) & =\frac{1}{2 !} e^{x-y} \\
W_{2}(x, y) & =\frac{1}{2 !} e^{-x+y} \\
& \vdots \\
U_{k}(x, y) & =\frac{(-1)^{k}}{k !} e^{x+y} \\
W_{k}(x, y) & =\frac{1}{k !} e^{x-y} \\
& =\frac{1}{k !} e^{-x+y}
\end{aligned}
$$




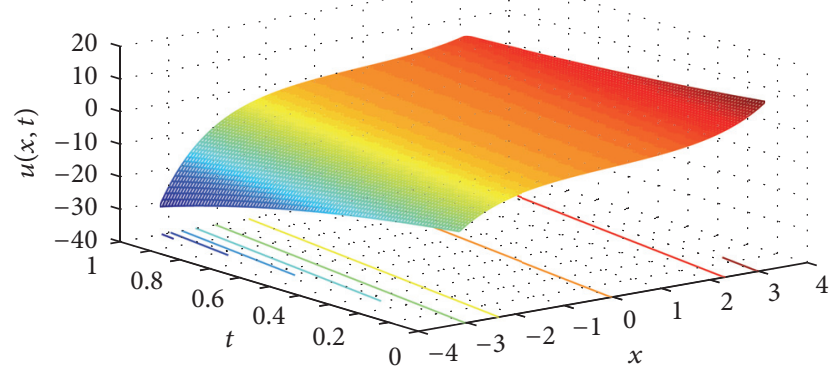

(a)

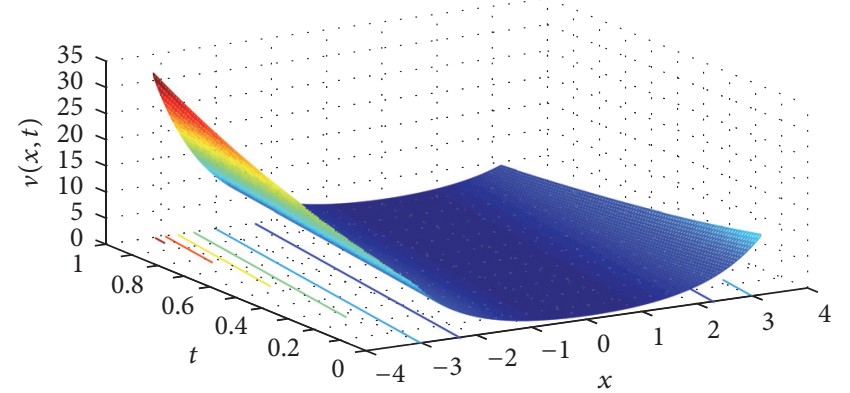

(b)

Figure 1: Behavior of $u$ (a) and $v$ (b) of initial value system (8) in $(-\pi, \pi)$.

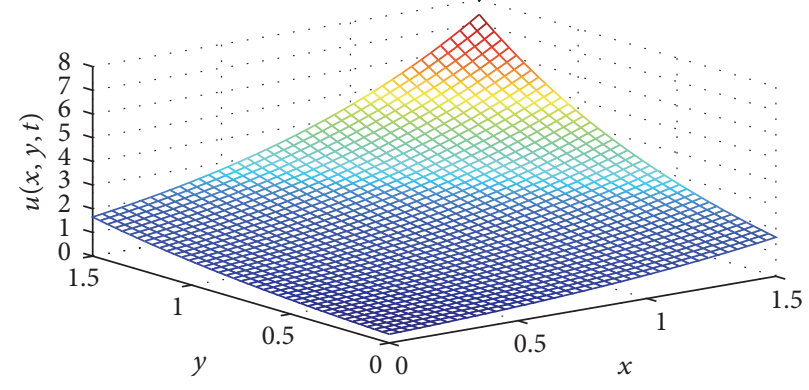

(a)

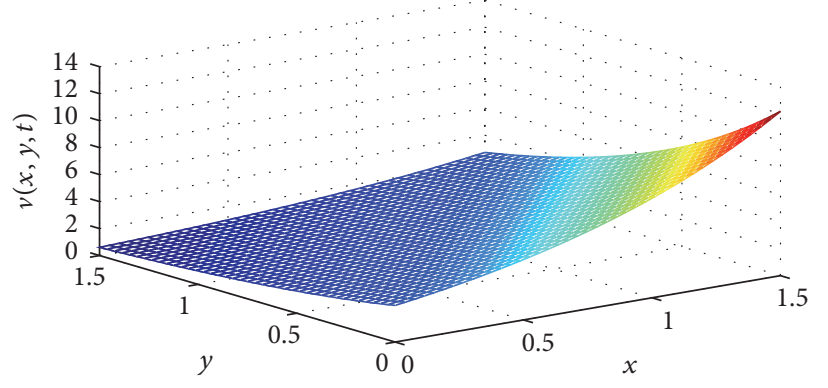

(b)

Figure 2: Behavior of $u$ (a) and $v$ (b) of initial value system (12) in $(0,1.5)$.

On using inverse RDT method (4), we get

$$
\begin{gathered}
u(x, y, t)=e^{x+y-t}, \\
v(x, y, t)=e^{x-y+t}, \\
w(x, y, t)=e^{-x+y+t} .
\end{gathered}
$$

This is the required exact solution of initial value system (12) of PDEs. The same solution is obtained by using homotopy perturbation method [21]. The solution behavior of $u, v$, and $w$ at $t=1$ is depicted in Figures 2 and 3.

Example 4. Consider the following form of IVS of twodimensional coupled viscous Burgers' equation:

$$
\begin{aligned}
\frac{\partial u}{\partial t} & =\frac{\partial^{2} u}{\partial x^{2}}+2 u \frac{\partial u}{\partial x}-\frac{\partial}{\partial x}(u v), \\
\frac{\partial u}{\partial t} & =\frac{\partial^{2} v}{\partial x^{2}}+2 v \frac{\partial v}{\partial x}-\frac{\partial}{\partial x}(u v), \\
u(x, 0) & =\sin x, \\
v(x, 0) & =\sin x .
\end{aligned}
$$

On using RDT method (16) reduces to a set of recurrence relations as follows:

$$
\begin{aligned}
& (1+k) U_{k+1}(x)=\frac{d^{2}}{d x^{2}} U_{k}(x) \\
& +\sum_{i=0}^{k}\left\{2 U_{i}(x) \frac{d}{d x} U_{k-i}(x)-\left(U_{i}(x) \frac{d}{d x} V_{k-i}(x)\right.\right. \\
& \left.\left.+V(x)_{i} \frac{d}{d x} U_{k-i}(x)\right)\right\}, \\
& (1+k) V_{k+1}(x)=\frac{d^{2}}{d x^{2}} V(x)_{k} \\
& +\sum_{i=0}^{k}\left\{2 V_{i}(x) \frac{d}{d x} V_{k-i}(x)-\left(U_{i}(x) \frac{d}{d x} V_{k-i}(x)\right.\right. \\
& \left.\left.\quad+V_{i}(x) \frac{d}{d x} U_{k-i}(x)\right)\right\} \\
& U_{0}(x)=\sin x, \\
& V_{0}(x)=\sin x .
\end{aligned}
$$

On solving system (17), we get

$$
U_{k}(x)=V_{k}(x)=\frac{(-1)^{k}}{k !} \sin x, \quad \forall k \geq 0 .
$$




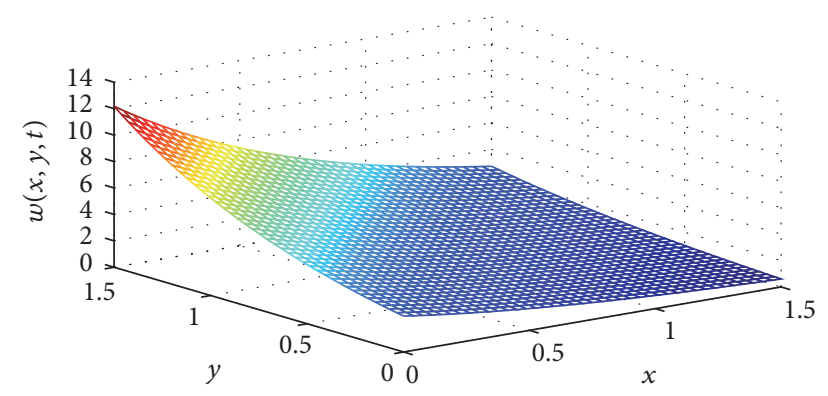

FIgURe 3: Behavior of $w$ of initial value system (12) in $(0,1.5)$.

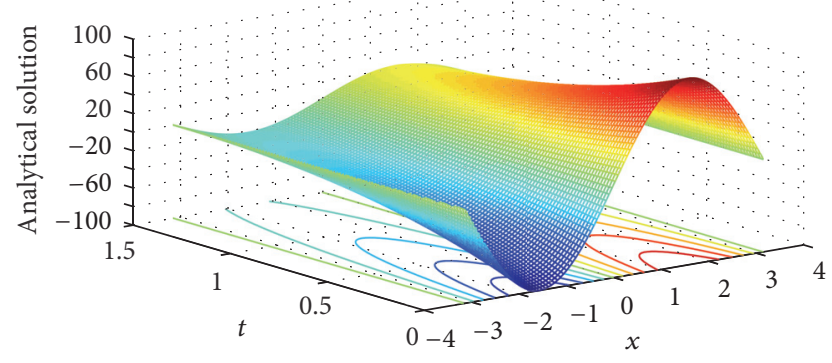

FIgURE 4: The behavior of $u$ and $v$ of initial value system of coupled viscous Burgers' equation (16) in $(-\pi, \pi)$ at different time levels.

On using inverse RDT method (4), we get

$$
\begin{aligned}
& u(x, t)=e^{-t} \sin (x), \\
& v(x, t)=e^{-t} \sin (x)
\end{aligned}
$$

This is the required exact solution of the initial value system of coupled viscous Burgers' equation (16). The same solution is obtained by homotopy perturbation method [21] and variational iteration method [22] for integer-order time derivatives. The physical behavior of $u, v$ is depicted in Figure 4.

Example 5. Consider the following form of initial value system of two-dimensional coupled viscous Burgers' equation:

$$
\begin{aligned}
\frac{\partial u}{\partial t} & =\frac{\partial^{2} u}{\partial x^{2}}+2 u \frac{\partial u}{\partial x}-\frac{\partial}{\partial x}(u v), \\
\frac{\partial u}{\partial t} & =\frac{\partial^{2} v}{\partial x^{2}}+2 v \frac{\partial v}{\partial x}-\frac{\partial}{\partial x}(u v), \\
u(x, 0) & =x^{2} \\
v(x, 0) & =x^{3} .
\end{aligned}
$$

On using RDT method (20) reduces to a set of recurrence relations as follows:

$$
\begin{aligned}
& (1+k) U_{k+1}(x)=\frac{d^{2}}{d x^{2}} U_{k}(x) \\
& +\sum_{i=0}^{k}\left\{2 U_{i}(x) \frac{d}{d x} U_{k-i}(x)-\left(U_{i}(x) \frac{d}{d x} V_{k-i}(x)\right.\right. \\
& \left.\left.+V(x)_{i} \frac{d}{d x} U_{k-i}(x)\right)\right\}, \\
& (1+k) V_{k+1}(x)=\frac{d^{2}}{d x^{2}} V(x)_{k} \\
& \quad+\sum_{i=0}^{k}\left\{2 V_{i}(x) \frac{d}{d x} V_{k-i}(x)-\left(U_{i}(x) \frac{d}{d x} V_{k-i}(x)\right.\right. \\
& \left.\left.\quad+V_{i}(x) \frac{d}{d x} U_{k-i}(x)\right)\right\}, \\
& U_{0}(x)=x^{2}, \\
& V_{0}(x)=x^{3} .
\end{aligned}
$$

Solving the recurrence relation (21), we get

$$
\begin{aligned}
& U_{1}(x)=2-5 x^{4}+4 x^{3} \\
& V_{1}(x)=6 x-5 x^{4}+6 x^{5} \\
& U_{2}(x)=\frac{1}{2} x\left(32-84 x+40 x^{3}-54 x^{4}-7 x^{5}\right) \\
& V_{2}(x)=\frac{1}{2} x^{2}\left(-84+168 x+6 x^{3}-77 x^{4}+96 x^{5}\right) ; \\
& U_{3}(x)=\frac{1}{3}\left(-96+384 x^{2}-908 x^{3}-225 x^{4}+336 x^{5}\right. \\
& \left.-679 x^{6}+276 x^{7}-\frac{261}{2} x^{8}\right) ; \\
& V_{3}(x)=\frac{1}{3}\left(-96+576 x+108 x^{3}-1995 x^{4}+3456 x^{5}\right. \\
& \left.\quad-21 x^{6}+380 x^{7}-\frac{2727}{2} x^{8}+1320 x^{9}\right) ; \\
& U_{4}(x)=\frac{1}{4}\left(320-2540 x-1128 x^{2}+4096 x^{3}\right. \\
& -\frac{39140}{3} x^{4}+7528 x^{5}-3108 x^{6}-\frac{21256}{3} x^{7} \\
& \left.+857 x^{9}-\frac{2981}{2} x^{10}\right) ;
\end{aligned}
$$




$$
\begin{aligned}
& V_{4}(x)=\frac{1}{4}\left(256 x-9912 x^{2}+28608 x^{3}-420 x^{4}\right. \\
& +10616 x^{5}-50764 x^{6}+62648 x^{7}-273 x^{8} \\
& \left.+\frac{23905}{3} x^{9}-\frac{43703}{2} x^{10}+17472 x^{11}\right) \\
& U_{5}(x)=\frac{1}{5}\left(-756+8000 x-51484 x^{2}+50472 x^{3}\right. \\
& \quad-22730 x^{4}-158174 x^{5}+\frac{353381}{3} x^{6}+52866 x^{7} \\
& \quad-\frac{638481}{4} x^{8}+\frac{354725}{6} x^{9}-\frac{31405}{2} x^{10} \\
& \left.+\frac{32349}{2} x^{11}-\frac{142103}{8} x^{12}\right) ; \\
& V_{5}(x)=\frac{1}{5}\left(-5532+47520 x-1812 x^{2}+71608 x^{3}\right. \\
& \quad-512430 x^{4}+880998 x^{5}-8771 x^{6}+319894 x^{7} \\
& +\frac{4389147}{4} x^{8}+\frac{2139285}{2} x^{9}-\frac{159665}{6} x^{10} \\
& +323167 \\
& 2
\end{aligned}
$$

Using inverse RDT method, the approximate solution is given as

$$
\begin{aligned}
& u(x, t)=U_{0}+U_{1} t+U_{2} t^{2}+U_{2} t^{3}+U_{4} t^{4}+U_{5} t^{5}+\cdots \\
& =x^{2}+\left(2-5 x^{4}+4 x^{3}\right) t+\frac{1}{2} x\left(32-84 x+40 x^{3}\right. \\
& \left.-54 x^{4}-7 x^{5}\right) t^{2}+\frac{1}{3}\left(-96+384 x^{2}-908 x^{3}\right. \\
& \left.-225 x^{4}+336 x^{5}-679 x^{6}+276 x^{7}-\frac{261}{2} x^{8}\right) t^{3} \\
& +\frac{1}{4}\left(320-2540 x-1128 x^{2}+4096 x^{3}-\frac{39140}{3} x^{4}\right. \\
& +7528 x^{5}-3108 x^{6}-\frac{21256}{3} x^{7}+3957 x^{8}+705 x^{9} \\
& \left.-\frac{2981}{2} x^{10}\right) t^{4}+\frac{1}{5}\left(-756+8000 x-51484 x^{2}\right. \\
& +50472 x^{3}-22730 x^{4}-158174 x^{5}+\frac{353381}{3} x^{6} \\
& +52866 x^{7}-\frac{638481}{4} x^{8}+\frac{354725}{6} x^{9}-\frac{31405}{2} x^{10} \\
& \left.+\frac{32349}{2} x^{11}-\frac{142103}{8} x^{12}\right) t^{5}+\cdots,
\end{aligned}
$$

$$
\begin{aligned}
& v(x, t)=V_{0}+V_{1} t+V_{2} t^{2}+V_{2} t^{3}+V_{4} t^{4}+V_{5} t^{5}+\cdots \\
& =x^{3}+\left(6 x-5 x^{4}+6 x^{5}\right) t+\frac{1}{2} x^{2}(-84+168 x \\
& \left.+6 x^{3}-77 x^{4}+96 x^{5}\right) t^{2}+\frac{1}{3}(-96+576 x \\
& +108 x^{3}-1995 x^{4}+3456 x^{5}-21 x^{6}+380 x^{7} \\
& \left.-\frac{2727}{2} x^{8}+1320 x^{9}\right) t^{3}+\frac{1}{4}\left(256 x-9912 x^{2}\right. \\
& +28608 x^{3}-420 x^{4}+10616 x^{5}-50764 x^{6} \\
& +62648 x^{7}-273 x^{8}+\frac{23905}{3} x^{9}-\frac{43703}{2} x^{10} \\
& \left.+17472 x^{11}\right) t^{4}+\frac{1}{5}\left(-5532+47520 x-1812 x^{2}\right. \\
& +71608 x^{3}-512430 x^{4}+880998 x^{5}-8771 x^{6} \\
& +319894 x^{7}-\frac{4389147}{4} x^{8}+\frac{2139285}{2} x^{9} \\
& \left.+228480 x^{13}\right) t^{5}+\cdots \\
& +159665 \\
& +10 \\
& +\frac{323167}{2} x^{11}-\frac{2687581}{8} x^{12}
\end{aligned}
$$

This is the required approximate solution of system (20). The approximate solution of system (20) is obtained by homotopy perturbation method [21] and variational iteration method [22] for integer-order time derivatives.

Example 6. Consider initial value system as follows:

$$
\begin{aligned}
\frac{\partial u}{\partial t} & =-\frac{\partial^{2} v}{\partial x^{2}}-2\left(u^{2}+v^{2}\right) v \\
\frac{\partial v}{\partial t} & =\frac{\partial^{2} u}{\partial x^{2}}+2\left(u^{2}+v^{2}\right) u \\
u(x, 0) & =\cos x \\
v(x, 0) & =\sin x .
\end{aligned}
$$

Setting $\psi=u+i v, i^{2}=-1$, then the above system can be written as

$$
\begin{aligned}
\frac{\partial \psi}{\partial t} & =i\left(\frac{\partial^{2} \psi}{\partial x^{2}}+2\left(|\psi|^{2}\right) \psi\right), \\
\psi(x, 0) & =e^{i x} .
\end{aligned}
$$


On using RDT method (25) reduces to a recurrence relation as follows:

$$
\begin{aligned}
& (1+k) \Psi_{k+1}(x)=i\left(\frac{\partial^{2} \Psi_{k}(x)}{\partial x^{2}}\right. \\
& \left.\quad+2 \sum_{k_{2}=0}^{k} \sum_{k_{1}=0}^{k_{2}} \bar{\Psi}_{k_{1}}(x) \Psi_{k_{2}-k_{1}}(x) \Psi_{k-k_{2}}(x)\right), \quad k \geq 1 \\
& \Psi_{0}(x)=e^{i x} .
\end{aligned}
$$

On solving the above relation, we get

$$
\begin{gathered}
U_{1}(x)=i e^{i x}, \\
U_{2}(x)=\frac{i^{2}}{2 !} e^{i x}, \\
\vdots \\
U_{k}(x)=\frac{i^{k}}{k !} e^{i x}
\end{gathered}
$$

The inverse RDT leads to

$$
\begin{aligned}
\psi(x, t) & =\sum_{k=0}^{\infty} \Psi_{k}(x) t^{k} \\
& =\left(1+i t+\frac{i^{2} t^{2}}{2 !}+\cdots+\frac{i^{k} t^{k}}{k !}+\cdots\right) e^{i x} \\
& =e^{i(x+t)} .
\end{aligned}
$$

This is the desired approximate solution of the initial value system (24), which is the same as that obtained in [28] using DTM.

\section{Concluding Remark}

In this paper, reduced differential transform method has been implemented successfully to five test problems of the initial value systems of time dependent partial differential equations including two-dimensional coupled viscous Burgers' equations. The obtained results agreed well with homotopy perturbation method [21], homotopy analysis method [3], variational iteration method $[6,22]$, and differential transform method [28]. Easiness and effectiveness are the strength of RDT method.

\section{Competing Interests}

The authors declare that there is no conflict of interests regarding the publication of this article.

\section{Acknowledgments}

Mahendra is very thankful to Babasaheb Bhimrao Ambedkar University, Lucknow, India, for the financial assistance to carry out the work.

\section{References}

[1] H. Jafari and V. Daftardar-Gejji, "Solving a system of nonlinear fractional differential equations using Adomian decomposition," Journal of Computational and Applied Mathematics, vol. 196, no. 2, pp. 644-651, 2006.

[2] K. C. Basak, P. C. Ray, and R. K. Bera, "Solution of nonlinear Klein-Gordon equation with a quadratic non-linear term by Adomian decomposition method," Communications in Nonlinear Science and Numerical Simulation, vol. 14, no. 3, pp. 718-723, 2009.

[3] H. Jafari and S. Seifi, "Solving a system of nonlinear fractional partial differential equations using homotopy analysis method," Communications in Nonlinear Science and Numerical Simulation, vol. 14, no. 5, pp. 1962-1969, 2009.

[4] S. E. Esipov, "Coupled Burgers equations: a model of polydispersive sedimentation," Physical Review E, vol. 52, no. 4, pp. 37113718, 1995.

[5] A. S. Bataineh, M. S. M. Noorani, and I. Hashim, "Approximate analytical solutions of systems of PDEs by homotopy analysis method," Computers \& Mathematics with Applications, vol. 55, no. 12, pp. 2913-2923, 2008.

[6] A.-M. Wazwaz, "The variational iteration method for solving linear and nonlinear systems of PDEs," Computers \& Mathematics with Applications, vol. 54, no. 7-8, pp. 895-902, 2007.

[7] Q. Wang and D. Cheng, "Numerical solution of damped nonlinear Klein-Gordon equations using variational method and finite element approach," Applied Mathematics and Computation, vol. 162, no. 1, pp. 381-401, 2005.

[8] M. A. Abdou and A. A. Soliman, "Variational iteration method for solving Burger's and coupled Burger's equations," Journal of Computational and Applied Mathematics, vol. 181, no. 2, pp. 245251, 2005.

[9] M. Dehghan, A. Hamidi, and M. Shakourifar, "The solution of coupled Burgers' equations using Adomian-Pade technique," Applied Mathematics and Computation, vol. 189, no. 2, pp. 10341047, 2007.

[10] H. P. Bhatt and A. Q. M. Khaliq, "Fourth-order compact schemes for the numerical simulation of coupled Burgers' equation," Computer Physics Communications, vol. 200, pp. 117138, 2016.

[11] M. Kumar and S. Pandit, "A composite numerical scheme for the numerical simulation of coupled Burgers' equation," Computer Physics Communications, vol. 185, no. 3, pp. 809-817, 2014.

[12] Y. B. He and X. H. Tang, "Numerical simulations of a family of the coupled viscous Burgers, equation using the lattice Boltzmann method," Journal of Statistical Mechanics: Theory and Experiment, vol. 2016, no. 2, Article ID 023208, 17 pages, 2016.

[13] C. A. J. Fletcher, "A comparison of finite element and finite difference solutions of the one- and two-dimensional Burgers' equations," Journal of Computational Physics, vol. 51, no. 1, pp. 159-188, 1983.

[14] P. C. Jain and D. N. Holla, "Numerical solutions of coupled Burgers' equation," International Journal of Non-Linear Mechanics, vol. 13, no. 4, pp. 213-222, 1978. 
[15] V. K. Srivastava and B. K. Singh, "A robust finite difference scheme for the numerical solutions of two dimensional time dependent coupled nonlinear Burgers' equations," International Journal of Applied Mathematics and Mechanics, vol. 10, no. 7, pp. 28-39, 2014.

[16] B. K. Singh and P. Kumar, "A novel approach for numerical computation of Burgers' equation in $(1+1)$ and $(2+1)$ dimensions," Alexandria Engineering Journal, 2016.

[17] G. Arora and B. K. Singh, "Numerical solution of Burgers' equation with modified cubic B-Spline differential quadrature method," Applied Mathematics and Computation, vol. 224, no. 1, pp. 166-177, 2013.

[18] B. K. Singh, G. Arora, and M. K. Singh, "A numerical scheme for the generalized Burgers-Huxley equation," Journal of the Egyptian Mathematical Society, vol. 24, no. 4, pp. 629-637, 2016.

[19] J. Liu and G. Hou, "Numerical solutions of the space- and timefractional coupled Burgers equations by generalized differential transform method," Applied Mathematics and Computation, vol. 217, no. 16, pp. 7001-7008, 2011.

[20] V. K. Srivastava, N. Mishra, S. Kumar, B. K. Singh, and M. K. Awasthi, "Reduced differential transform method for solving (1 $+\mathrm{n}$ )-Dimensional Burgers' equation," Egyptian Journal of Basic and Applied Sciences, vol. 1, no. 2, pp. 115-119, 2014.

[21] A. Yildirim and A. Kelleci, "Homotopy perturbation method for numerical solutions of coupled Burgers equations with time- and space-fractional derivatives," International Journal of Numerical Methods for Heat \& Fluid Flow, vol. 20, no. 8, pp. 897909, 2010.

[22] A. Prakash, M. Kumar, and K. K. Sharma, "Numerical method for solving fractional coupled Burgers equations," Applied Mathematics and Computation, vol. 260, pp. 314-320, 2015.

[23] Y. Keskin and G. Oturanç, "Reduced differential transform method for partial differential equations," International Journal of Nonlinear Sciences and Numerical Simulation, vol. 10, no. 6, pp. 741-749, 2009.

[24] J. Yu, J. Jing, Y. Sun, and S. Wu, “( $n+1)$ dimensional reduced differential transform method for solving partial differential equations," Applied Mathematics and Computation, vol. 273, pp. 697-705, 2016.

[25] B. K. Singh and P. Kumar, "FRDTM for numerical simulation of multi-dimensional, time-fractional model of Navier-Stokes equation," Ain Shams Engineering Journal, 2016.

[26] B. K. Singh and V. K. Srivastava, "Approximate series solution of multi-dimensional, time fractional-order (heat-like) diffusion equations using FRDTM," Royal Society Open Science, vol. 2, Article ID 140511, 2015.

[27] V. K. Srivastava, S. Kumar, M. K. Awasthi, and B. K. Singh, "Two-dimensional time fractional-order biological population model and its analytical solution," Egyptian Journal of Basic and Applied Sciences, vol. 1, no. 1, pp. 71-76, 2014.

[28] A. S. V. Ravi Kanth and K. Aruna, "Two-dimensional differential transform method for solving linear and non-linear Schrödinger equations," Chaos, Solitons and Fractals, vol. 41, no. 5, pp. 2277-2281, 2009. 


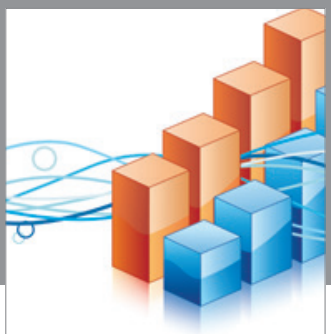

Advances in

Operations Research

vatem alat4

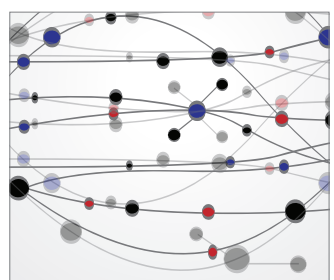

\section{The Scientific} World Journal
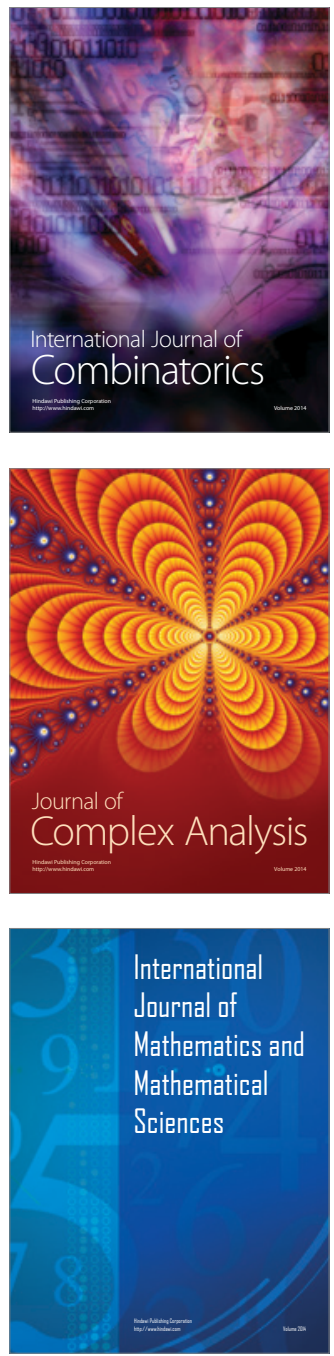
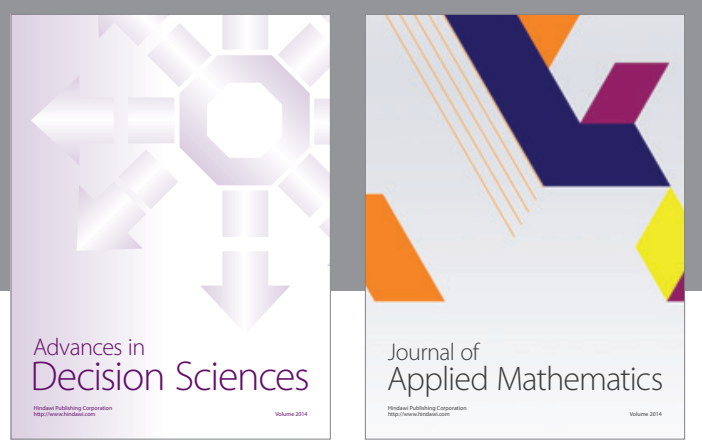

Algebra

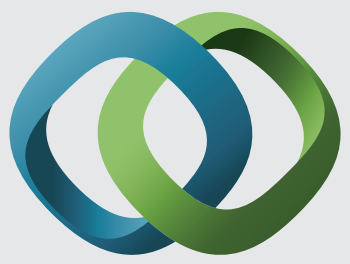

\section{Hindawi}

Submit your manuscripts at

http://www.hindawi.com
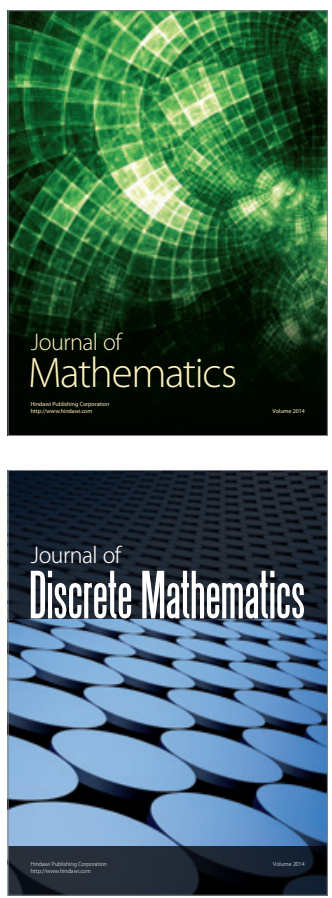

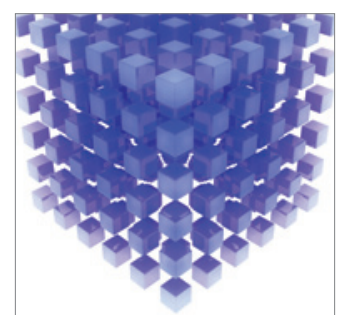

Mathematical Problems in Engineering
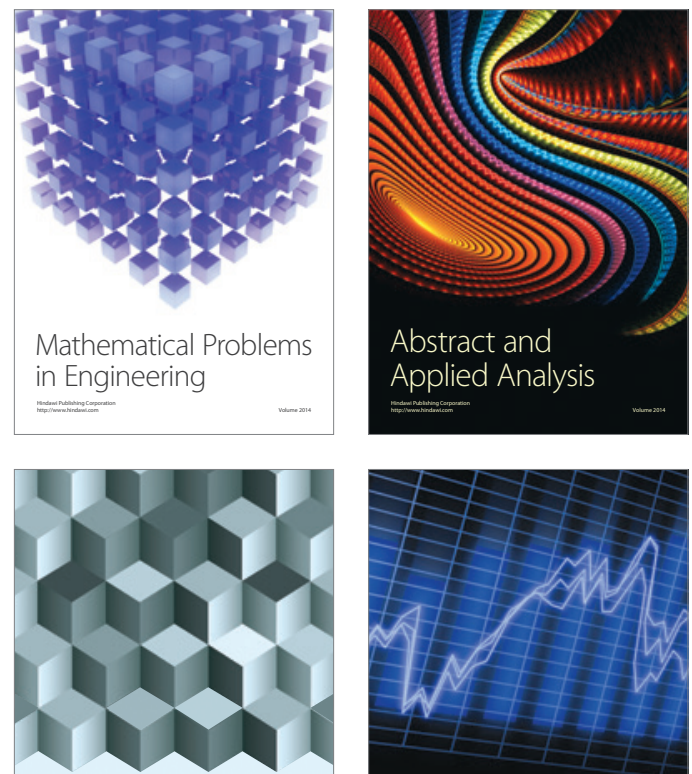

Journal of

Function Spaces

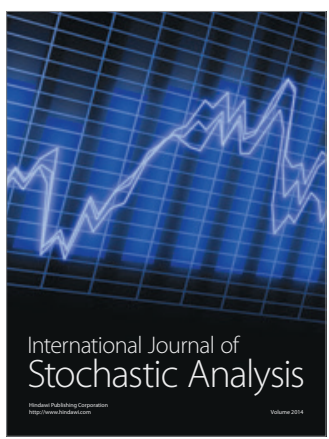

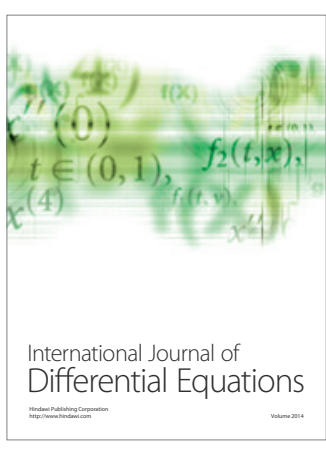
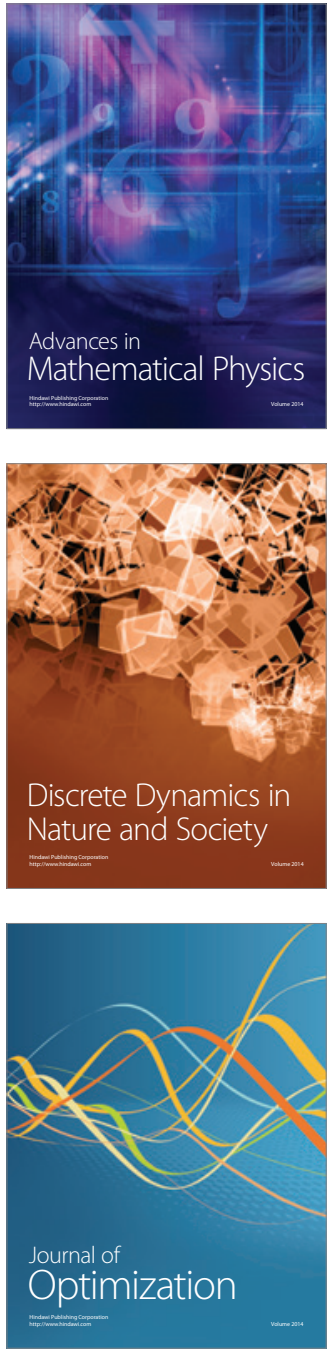